\title{
Maternal or neonatal infection: association with neonatal encephalopathy outcomes
}

Meike Jenster, Sonia L. Bonifacio, Theodore Ruel, Elizabeth E. Rogers, Emily W. Tam, John Colin Partridge, Anthony James Barkovich, Donna M. Ferriero and Hannah C. Glass

Correction to: Pediatric Research (2014) 76, 93-99 doi:10.1038/pr.2014.47

In this article, a single subject was reported as having "Group B streptococci" (GBS) infection, whereas this should have read "Group A streptococci" (GAS) as a re-review of the data indicate a Streptococcus viridans infection. 